www.jmscr.igmpublication.org

Impact Factor 5.84

Index Copernicus Value: 83.27

ISSN (e)-2347-176x ISSN (p) 2455-0450

crossref DOI: _https://dx.doi.org/10.18535/jmscr/v5i3.89

Journal Of Medical Science And Clinical Research

\title{
Comparative Effect of Kinesiotape and Rigid Tape with Therapeutic Putty Exercise on Pinch Strenght in Instrumentalists - A Randomized Clinical Trial
}

\author{
Authors \\ Dhaval Chivate MPT ${ }^{1}$, Samrudhdi Satavase BPT ${ }^{2}$, Savita Boragi BPT ${ }^{3}$ \\ ${ }^{1}$ Lecturer, Department of Sports Physiotherapy, Kleu Institute of Physiotherapy \\ Email:d_chivate@yahoo.com \\ ${ }^{2,3}$ Intern, KLEU Institute of Physiotherapy
}

\begin{abstract}
Study objectives- The purpose of this study was to investigate the comparative effect of kinesio tape and rigid tape with therapeutic putty exercises on pinch strength in instrumentalists.

Design- Comparative study

Settings- All the subjects were included from all music schools of Belgaum city.

Method- Total of 30 subjects was recruited for study on the basis of inclusion and exclusion criteria after signing the informed consent form. Subjects were divided into two groups ( $A=$ Kinesio tape and therapeutic putty exercises and $B=$ Rigid tape and therapeutic putty exercises), Kinesio tape and rigid tape application was done on painful hand following which pinch strength and VAS (Visual analogue scale) was measured of affected hand pre and post intervention. Kinesio taping was done for 2-3 days and Rigid tape was applied for 2 days along with therapeutic putty exercises done for 3 weeks with 3 sessions per week for 10-15 min with progressive repetition and measurements were taken again of treated hand.

Outcome Measure- Visual analogue scale and pinch gauge dynamometer.

Results- The result showed that there was a significant change in group A ieKinseio tape and therapeutic putty exercises as compared to group B ie Rigid tape and therapeutic putty exercises and there was significant change in pain in both the groups with reference to pinch strength in instrumentalist.

Conclusion- The results suggest that application of Kinesio tape along with therapeutic putty exercises has effect on pain and pinch strength in instrumentalist. Further studies involving a larger sample of subjects and a same condition are warranted to confirm our findings.

Keywords- Kinesio tape, Rigid tape, Therapeutic putty exercises, Pinch strength, instrumentalist.
\end{abstract}

\section{Introduction}

Music is a form of art and cultural activity which has silence as a medium along with sound. Music is a combination of vocal techniques and use of instruments and performed with help of this both. It is an extraordinary piece made up of instruments and singing ${ }^{1}$ Musicianare also known as instrumentalist. Musicians are specialized in different variety of styles which includes different skills which are performed by professional by conducting, arranging, composing and singing of music $^{2}$. Instrumentalists are similar like athletes. They also are prone to musculoskeletal injuries like athletes. These injuries can also end their 
career or affect their life and may cause irreversible damage due to which successful return to musical activity may not be possible ${ }^{3}$. Generally musicians start playing at very early age as they grow up they actually become anatomically shaped to their instrument ${ }^{4}$. In $19^{\text {th }}$ century physicians started recognizing a condition like "musician's cramp" as similar to "writer's cramp". Also there has been research on condition known as "pianist's breakdown" which resembles "overuse syndrome"5. A mild carpal tunnel syndrome typist may still adequately type but a first chair violinist with the same condition may lose that position due to inability to play violin .Every year about $(76 \%)$ musicians suffer from problems which affect their performance. The common conditions seen in musicians are overuse syndrome, radial, median and ulnar neuropathy, cervical radiculopathy, thoracic outlet syndrome, focal dystonia, joint hyper mobility, Linburg Comstock syndrome, Dequervain's syndrome and carpal tunnel syndrome ${ }^{4 .}$

It is also seen that pinch strenght is very important in instrumentalists. Usually musicians have weaker pinch strength compared to non musicians. It is seen more common in hand which is used while playing and tip pinch is more affected due to repetitive use of thumb and index finger. ${ }^{6}$ Several factors may be involved in the decreased pinch strength in a musician, including a sudden increase in playing time, practicing for long hours without taking breaks and improperly maintained instrument. ${ }^{7}$ Keyboard and string players have more decreased pinch strength compared to other instrumentalist. Every year 10\%-15\% musicians are suffer from wrist injuries which reduce their pinch strength. ${ }^{8}$ There is lack of research work done in physiotherapy management for wrist pain in instrumentalists. Kinesio-taping is very useful in rehabilitation. KT tape is a specialized tape made up of elastic cotton fibre and acrylic adhesive, in a wavy pattern. . KT can be applied to reduce pain and relieve muscle spasm, it also helps to increase proprioception and strengthen weak muscles. KT may also be beneficial in improving strength ${ }^{9}$. Application of KT can facilitate muscle strength by pulling onto the fascia due to its elastic property ${ }^{10}$.

Rigid tape is used to stabilize a joint and also to limit movements across it. Rigid tapes help to prevent joint and ligament injury during a sport, as well as it helps to protect and stabilize a previously injured joint. Rigid tape provides physical barrier to movements, as well as it enhances proprioception and muscle function. ${ }^{11}$ As a therapy, putty is useful in the treatment carpal tunnel syndrome, focal dystonia, trigger finger, hand weakness, case of tendon surgery or in case of hand fracture. Therefore, putty hand exercises help to improve the overall hand strength. Different ways to improve hand and finger's strength include using a towel or an exercise tool like Digi Flex for improvement of hand strength. These exercise can be performed in a simple manner by rolling the putty in between the hand followed by squeezing it in between the fingers and maintaining the tension for 3-5 seconds and repeating it for 15-20 times. ${ }^{12}$

\section{Method}

Total 30 male and female subjects with an age group 21-45 year were recruited for the study. They were recruited from various music schools around Belagavi city. Subjects were selected and assigned by convenient sampling and randomized distribution through envelope method. The subjects signed an informed a consent form following which they were randomly assigned to two groups with 15 subjects in Group A and 15 subjects in Group B. Inclusion ciriteria Instrumentalist of aged 21-45 years, Practicing for two or more than two years and Instrumentalist practising for three or more than three hours per week. Exclusion criteria- Subjects who have undergone any upper limb nerve injury and With recent hand and wrist injuries in last 6 months. Group A comprising of 15 subjects received Kinesio tape and therapeutic putty exercises. Patient is in sitting position with wrist, forearm 


\section{JMSCR Vol||05||Issue||03||Page 18833-18841||March}

and elbow should be maintained in full flexion, full pronation and full extension. An I- shaped kinesio tape (a single strip) is applied for facilitation technique with rounded edges. The tape will be applied on extensor muscle group from origin to insertion. Followed by use of therapeutic putty exercises for 10-15 repetitions of each exercise with progression. Measurements were taken before applying tape and post three weeks according to visual analogue scale(VAS) and pinch gauge dynamometer. Followed by alone use of therapeutic putty exercises. Three sessions per week for three week of putty. Group B also comprising of 15 subjects received rigid tape and therapeutic putty exercises and application of tape was in same manner as group A. The therapeutic exercises which were used was, the patient will be sitting in comfortable position in chair with lowbacked, firm seated with tape applied to the forearm. Patient is then ask to squeeze the putty with hand, move the putty between the wrist and finger tips, place the wrist properly in putty and then fingers are stretch and thumb and index shaped in putty for 10-15 repetitions of each exercise with progression following 3 weeks measurements were taken.
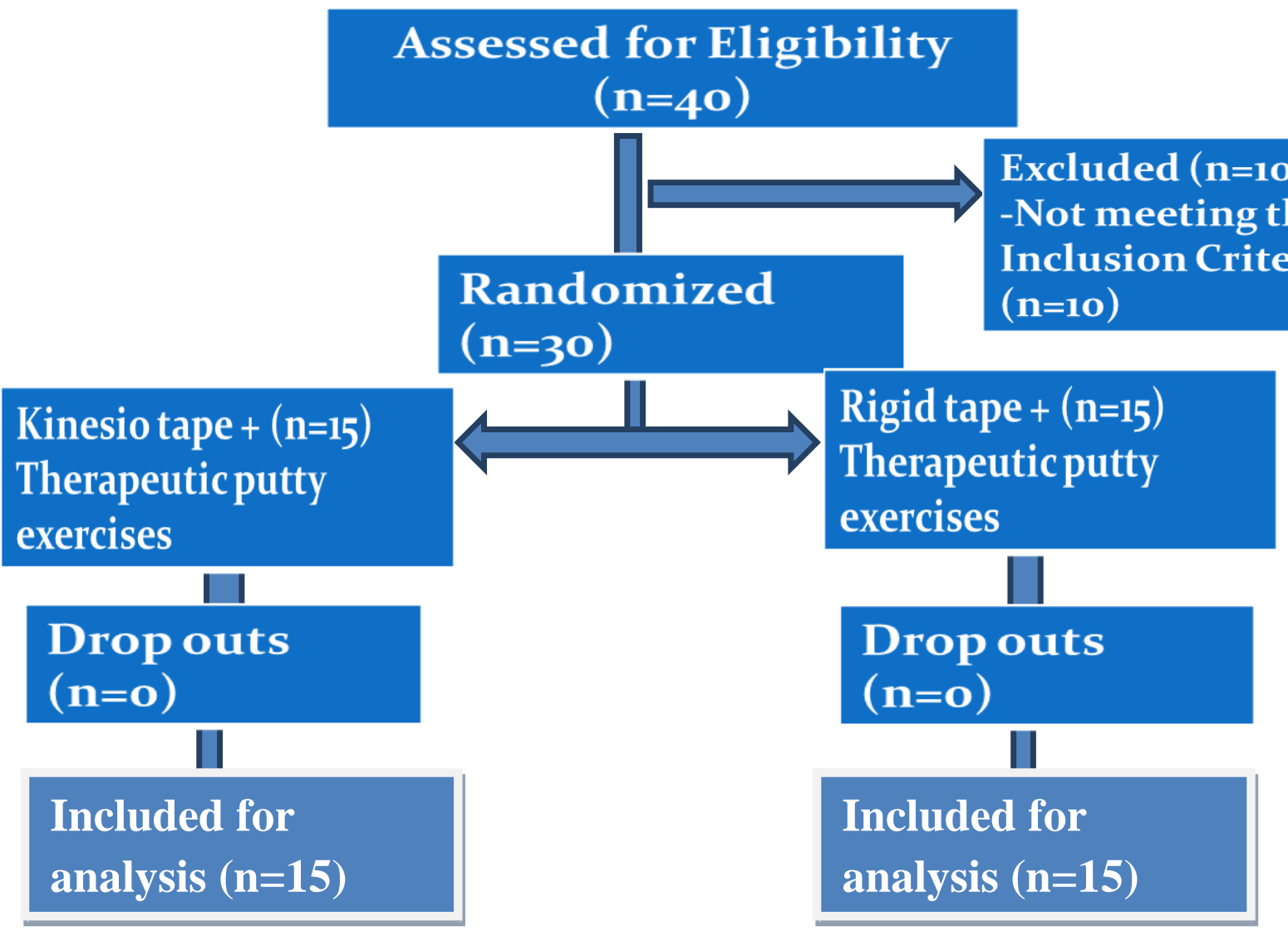

\section{Results}

- Data analysis was done using the SPSS 16.0 version.

- Mann-Whitney U test was done to compare pre and post intervention reading of VAS and Pinch Gauge Dynamometer (kgs) within group A\&B.

- Wilcoxon matched pairs test was done to compare pre and post intervention reading of VAS and Pinch Gauge Dynamometer (kgs) between the group A\&B. 


\section{JMSCR Vol||05||Issue||03||Page 18833-18841||March}

\section{Annexure}

Comparison of Two Study Groups (Group A And Group B) With Respect to Vas Scores at Pretest and Posttest Time Points By Mann-Whitney U Test

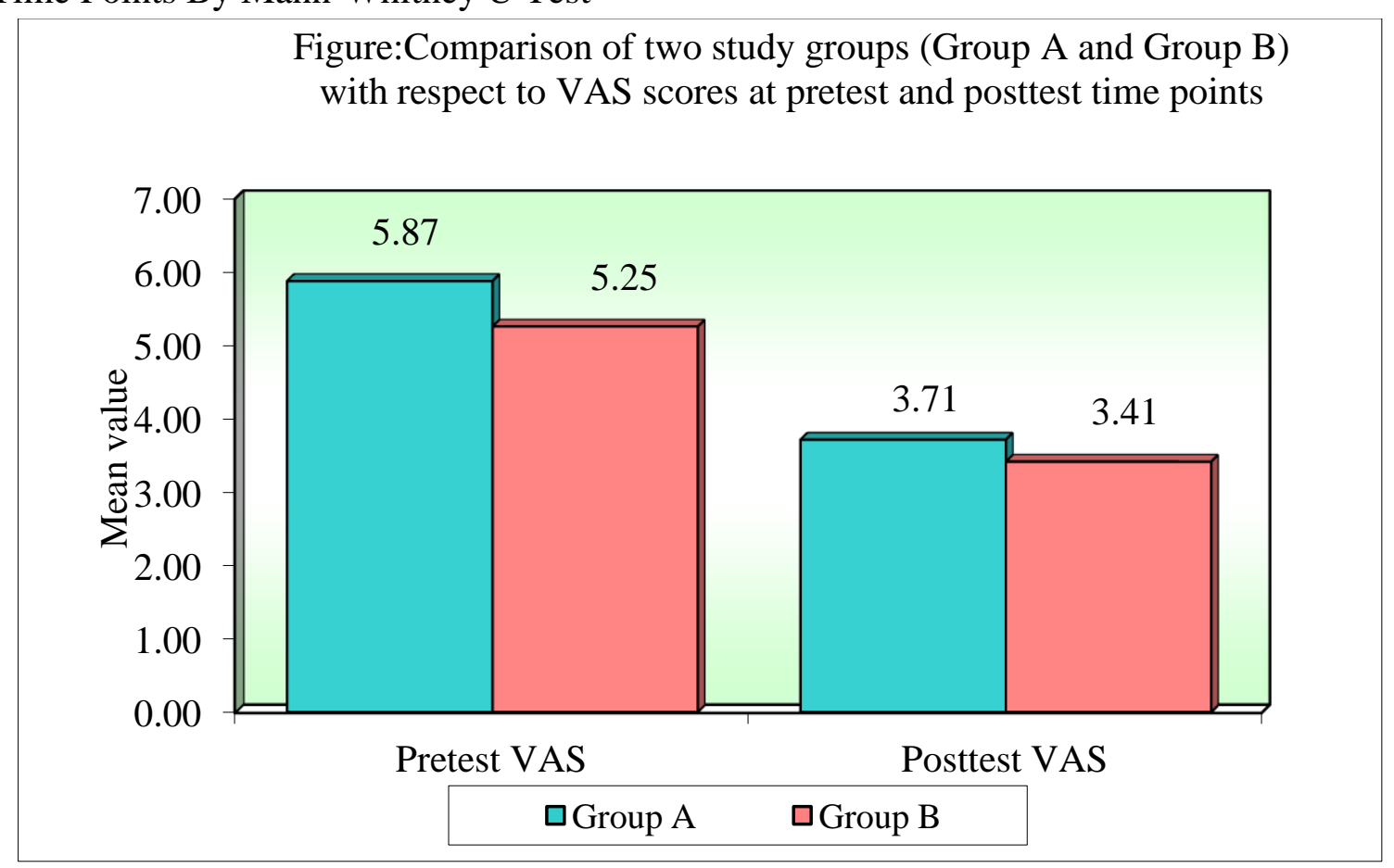

Comparison of Pretest and Posttest Vas Scores in Two Study Groups (Group A And Group B) By Wilcoxon Matched Pairs Test

\begin{tabular}{|c|c|c|c|}
\hline \multicolumn{1}{|c|}{ Figure:Comparison of pretest and posttest VAS scores in two study groups } \\
(Group A and Group B)
\end{tabular}




\section{JMSCR Vol||05||Issue||03||Page 18833-18841||March}

Comparison of Two Study Groups (Group A And Group B) with Respect to Pinch Gauge Dynamometer (KGS) Scores at Pretest and Posttest Time Points by Mann-Whitney U Test

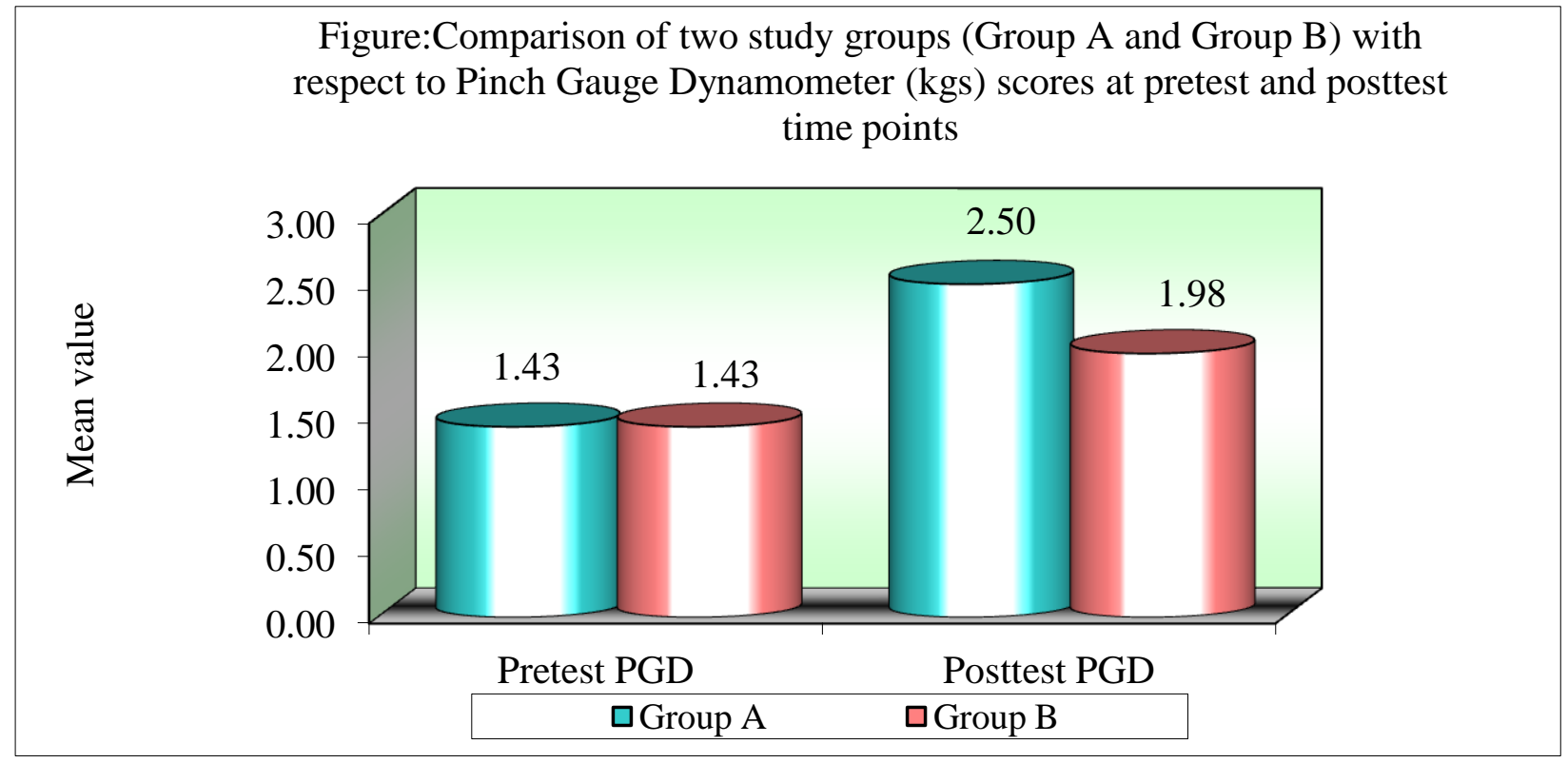

Comparison of Pretest and Posttest Pinch Gauge Dynamometer (Kgs) Scores in two Study Groups (Group A And Group B) By Wilcoxon Matched Pairs Test

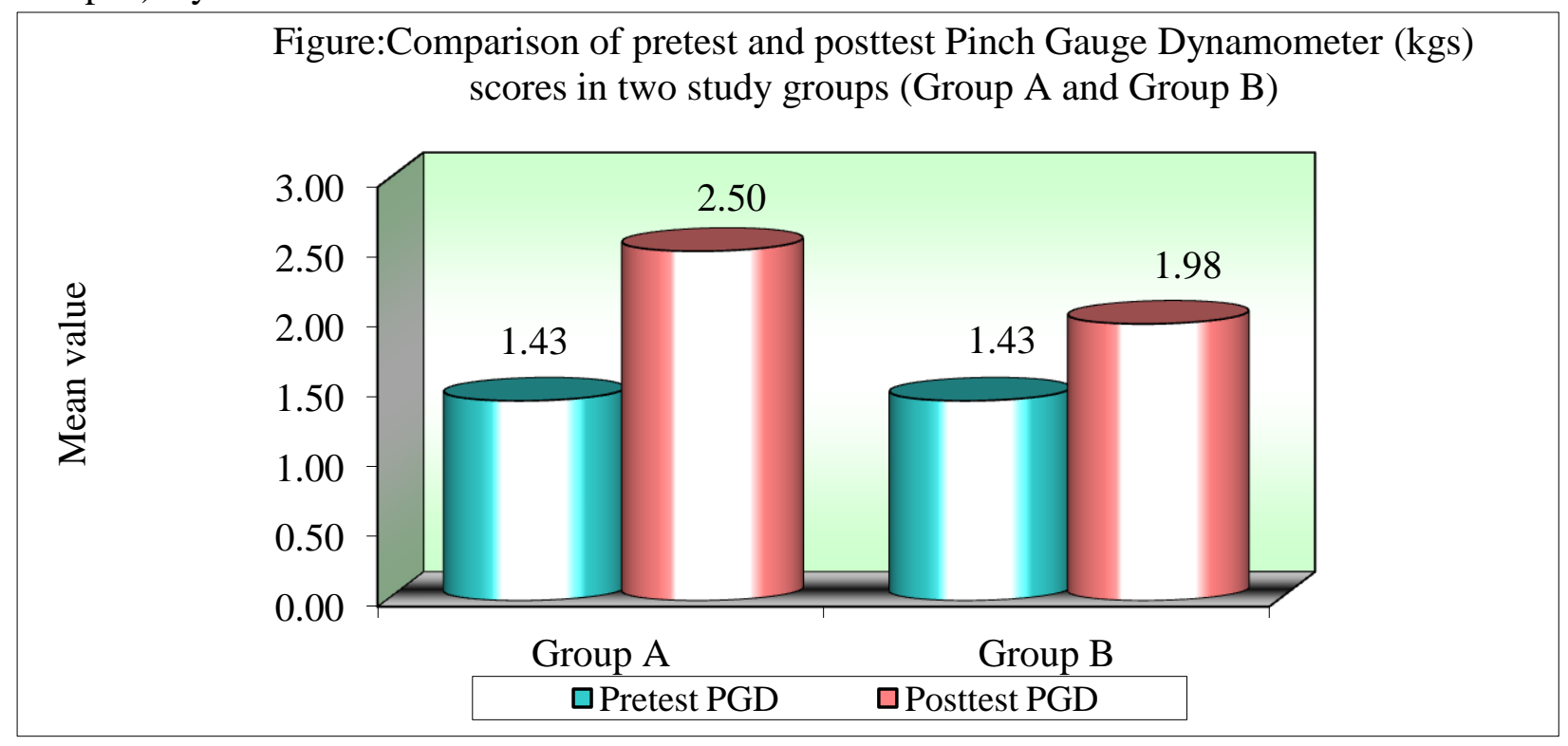

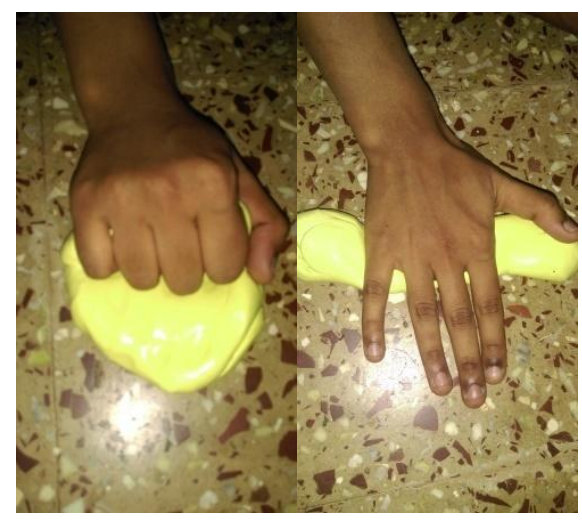

Figure (1)

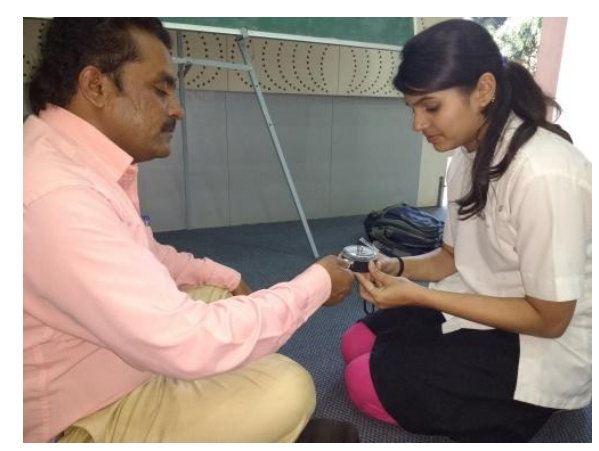

Figure( 2) 


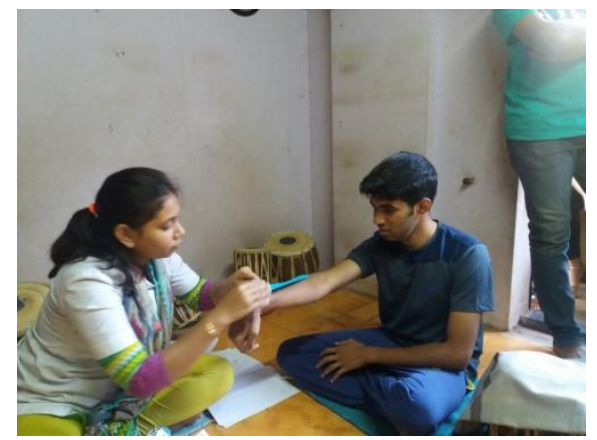

Figure (3)

(1)Subject performing putty exercises. (2)

Checking pinch strength. (3) Application of tape.

\section{Discussion}

The result supports the hypothesis that Kinesio tape and rigid tape in instrumentalist with therapeutic putty exercises helps to increase the pinch strength and reduce pain in wrist.

The present study revealed male dominance in both the groups. (group A $86.67 \%$ males whereas $60 \%$ in group B). Study done by Carl Z, Helena B, Jenny $\mathrm{K}$ et al., on Musculoskeletal problems among male and female music students proved that females were more affected than males in terms of body parts affected and level of stress ${ }^{13}$. The results of this study are contradictory to the results of the present study. This can be due to absence of homogeneity in the study samples in both the groups.

A study done by Susan EG Sims and Laura Engel et al on 200 subjects with age range 18-28years demonstrated that male musicians have weaker pinch and grip strength compared to male nonmusicians and female musicians have also weaker pinch and grip strength compared to female nonmusicians ${ }^{6}$. Another study done on the incidence of hand problems in music students showed that the mean age was 21.5 years suggesting that the younger age group is more prone to hand and wrist problems ${ }^{15}$. In present study the age range was 21-45years, as in this age group musculoskeletal injuries are more common in musicians due to continuous practice and repetitive movements which causes degenerative changes in the part.
The results showed that females have weaker strength compared to males when it was compared in both groups. A study done by Gavin Miller and Fiona peck showed that right hand dominance is more in musicians also it is more prone to injuries as compared to left hand but the results were not statistically significant, similar results were seen in this study when hand dominance was considered and it showed right hand dominance in both the groups as compared to left hand, but the results were not statistically significant ${ }^{14}$.

In present study VAS score was taken before and after intervention, with the help of horizontal scale marked from $0-10 \mathrm{~cm}$ scale on another paper and recorded in assent form. Intergroup $\mathrm{p}$ value for VAS score was statistically considered. Intra group $\mathrm{p}$ value for both the groups A and B were statistically significant. The effects of kinesio tape and therapeutic putty exercises (GroupA) were significant with $\mathrm{p}$ value for pinch strength and VAS score respectively. The gate control theory can be one possible explanation for reduction in pain as it has being proposed that neuromuscular pathway are stimulated via increase afferent feedback and cutaneous stretch from the application of kinesio tape $\mathrm{e}^{23,24}$. It limits the motion in the damaged tissues thus promoting healing and provides awareness to the subject regarding movement of the hand. Significant results were also seen for group B where rigid taping was applied along with therapeutic putty exercises.

A study conducted by Wircha Mishra and Amitabha De on 85 tabla players where symptoms of discomfort were recorded. VAS was considered as outcome measures to check musculoskeletal problems in tabla players and results showed VAS scores were more significant in the right shoulder and although wrist was clinically significant, but statistically it wasn't proved ${ }^{16}$. Similar study was done by Eri Yoshimora and Pamela Mia Paul to assess the level of pain in piano players considering VAS to be the outcome measure ${ }^{17}$. In the present study, involvement of the wrist and hand in music students proved its statistical significance. 
In the current study, within group measurement of pinch strength was taken and compared pre and post intervention in each group was statistically significant with $\mathrm{p}$-value for both the groups. The cause for increase in pinch strength could be due to effect of therapeutic putty exercises which strengths hand muscles and also because of application of tape which caused reduction in wrist pain that might have induced the changes in pinch strength. Between group measurements for pinch strength was statistically significant post treatment, and the difference was found out to statistically significant.

Comparison of outcome measures between both the groups showed that kinesio taping and therapeutic putty exercise was significantly better than rigid taping and therapeutic putty exercises. A study done by Sofia Brorsson and Maritan villiges et al on hand function in rheumatoid arthritis patients with six weeks hand exercise (therapeutic putty exercises) program showed significant improvement in hand function post six weeks on force measurements and hand function. Extension force and flexion force increased at the end of 12 weeks $^{18}$. In the present study, the result clearly depicted that there was more marked increase in pinch strength with group A (KT), as compared to group B (RT) which showed group A improved more than group B. Therapeutic putty exercises done along with taping provides greater awareness of the movement thereby a higher muscular activation is generated which provides training effects on the muscles of the forearm and hand.

Studies have shown that kinesio tape has proved to be effective in increasing pinch strength and the possible reason for this could be that while applying the kinesio tape there is longitudinal stretch applied on muscles of the forearm and hand which activates and strengthens the weaker muscles by pulling onto fascia due to its elastic property. I-shape kinesio and rigid taping was done in group A and B respectively for the wrist extensor muscles in the present study. Specific taping resulted in greater tactile stimulation of the wrist extensors and inhibition of other muscles ${ }^{20}$. Improvement in strength of the wrist extensors placed the wrist and hand in a mechanical advantage position thereby improving the pinch strength in the subjects. A similar study was done by Bill Vicenzio and Jane Brooksbank to see the effect of rigid tape on elbow for pain-free grip strength on lateral epicondylgia and the result showed a significant change with pain free grip strength in subjects after using rigid tape with statistically significant $\mathrm{p}$-value ${ }^{19}$.

To our knowledge there was no treatment regimen done on musicians for the pain and reduced pinch strength. So the present study was done following a proper treatment regimen considering various studies done previously. A meta analysis study was done by Robert Csapo on effects of kinesio taping on skeletal muscle strength on 19 studies, on 538 subjects, the overall population subjects showed that on average the potential to increase strength by application of KT is negligible ${ }^{21}$. One more study was done by Amir Buljina and Mihra Taljanovic showed that use of balance resistive hand exercises using therapeutic putty for treatment of rheumatoid hand helps in improvement of functional status of hand following three weeks ${ }^{22}$. Therefore taking in consideration different studies, in our study three weeks intervention programme was carried out where application of tape was done for 2 days following which three sessions per week for three weeks of therapeutic putty exercises was given and measurements were taken pre and post treatment on VAS and Pinch Gauge meter. Following a standard exercise and taping regimen helped in greater improvement in outcome as well as subject satisfaction.

To the best of our knowledge this is the first clinical trial to demonstrate the effectiveness of kinesio tape versus rigid tape with therapeutic putty exercises in instrumentalists.

According to the above stated discussion no study has been conducted, so a comparison has been made between the effectiveness of kinesio tape and therapeutic putty exercises versus rigid tape 
and therapeutic putty exercises to reduce pain and improve pinch strength in instrumentalists within the age group of 21-45 years. Thus taking into consideration the statistical analysis of the study we can conclude that both kinesio tape and rigid tape along with therapeutic putty exercises reduces pain and increase pinch strength in instrumentalists but, kinesio tape and therapeutic putty exercises, more significantly reduces pain and increases pinch strength than compared to rigid tape and therapeutic putty exercises.

Thera putty exercises thus help in preventing injuries in instrumentalists also can be used as a protocol to prevent injuries and to increase in performances.

\section{Limitation}

1) Long term effect of taping was not assessed

2) Study sample was not homogenous in terms of gender.

3) Small sample size.

\section{Conclusion}

The present study concluded that both the technique is effective in improving the pain and pinch strength in instrumentalists. The study highlights that kinesio tape and therapeutic putty exercises is better choice of treatment in improving pain and pinch strength in instrumentalist than rigid tape and putty exercises.

\section{Strength of the Study}

Based on the result of this study we came to know about the effect of kinesio tape and therapeutic putty and observed the changes in pain and pinch strength related with the instrumentalists. In both groups both the techniques showed independent effectiveness in improving pinch strength. Depending on results we can emphasise that the effect of kinesiotape and therapeutic putty proved more effective for treating the instrumentalists with pain and reduce pinch strength than rigid tape.

\section{References}

1. Music. [Homepage On The Internet]. No Date [Cited 2016 June 8]. Available From: Https://En.Wikipedia.Org/Wiki/Music

2. Musician. [Homepage On The Internet]. No Date [Cited 2016 June 8]. Available From:

Https://En.Wikipedia.Org/Wiki/Musician

3. S.Sheibani-Rad, S.Wolfe, J. Jupiter. Hand Disorders In Musicians. 2013:Vol 95-B No 2, 1.

4. Fadi Joseph Bejjani, Glenn M. Kaye, Melody Benham. Musculoskeletal And Neuromuscular Conditions Of Instrument Musicians. Arch Phys Med Reh 1996: 77:406-13.

5. Fry Hjh. Overuse Syndrome In Musicians100 Years Ago. Med J Aust 1986; 145:620-5.

6. Susan Sins, Laura Engel, Warren Hammel, John Elfar. Hand Sensibility Strength And Laxity Of High Level Musicians Compared To Non Musicians. The Journal Of Hand Surgery. Vol 40, Issue 10. 15.1996-2002.

7. Barbara Robinson. Rehabilitation Of Cellist After Surgery ForDequervain's Tenosynovitis And Intersection Syndrome. Medical Problems Of Performing Artists.18-3(2006)106.

8. Joseph F Slade, John Mahoney, John Bailinger. Wrist And Hand Injuries In Musicians- Management And Prevention. The Journal Of Musculoskeletal Medicine. 16.9(1999)542.

9. Kase K, Tatsuyuki H, Tomoko O :Kinesio Taping Perfect Manual. Usa: Universal Printing And Publishing, 1996.

10. Vithoulka I, Beeka A, Malliou P, Et Al : The Effects Of Kinesio Taping On Quadriceps Strength During Isokinetic Exercise In Healthy Non Athlete Women. IsokinetExercSci, 2010, 18: 1-6.

11. Rigid Tape [Homepage On The Internet]. No Date [Cited 2016 June 8]. Available 
From:

Http://Www.Apemedical.Com.Au/Shop/Li stings/Rigid-Strapping-Tapes/

12. Brett Sears. Exercises You Can Do With Therapy Putty. Feb 28: 2015.

13. Zetterberg C, Backlund H, Karlsson J, Werner H, Olsson L. Musculoskeletal problems among male and female music students. Medical Problems of Performing Artists. 1998 Dec 1;13:160-6.

14. Gavin Miller, Fiona Peck, Anne Brain, Stewart Watson. Musculotendious anomalies In Musician And Non Musician Hands. Musculotendious Anomalies.2003. Vol.112, No.7.

15. Manchester RA. The incidence of hand problems in music students. Organ. 1988;3(1):16-8.

16. Wricha Mishra, Amitabha De, Somanth Gangopadhyay, Ananga M Chandra. Playing-Related Musculoskeletal Disorders Among Indian Table Players. Medical Problems Of Performing Artists. Jun 2013:107-111.

17. Eri Yoshimura, Pamela Mia Paul, CyrielAerts, Kris Chesky. Risk Factors For Piano-Related Pain Among College Students. Medical Problems Of Performing Artists.Sep 2006;21:118-215.

18. Sofia Borosson, MaritaHilliges, Christer Sollerman, Anna Nilsdotter. A Six Week Hand Exercise Programme Improves Strength And Hand Function In Patients With Rheumatoid Arthritis. Rehabil Med 2009; 41: 338-342.

19. Bill Vicenzio, Jane Brooksbank, Joanne Minto, Sonia Offord, AatitPaungmali. Intial Effect Of Elbow Taping On PainFree Grip Strength And Pressure Pain Threshold. JornalOf Orthopaedic And Sports Physical Therapy. Jul 2003:Vol 33. No.7.
20. Yi-Liang Kuo, Yueh-Chu Huan: Effects Of The Application Direction Of Kinseio Taping On Isometric Muscle Strength Of The Wrist And Fingers Of Healthy AdultsA Pilot Study. J.Phys. Ther. Sci.2013, 25:287-291.

21. Robert Csapo, Luis M. Alergre. Effects OfKinseio Taping On Skeletal Muscle Strength- A Meta-Analysis Of Current Evidence. Journal of Science And Nedicine In Sport; Jul 2015: 18:450-456.

22. Amir Bulijna, MihraTaljanovic, Dijana Advice, Tim Hunter. Physical And Exercise Therapy For Treatment Of The Rheumatoid Hand. Arthritis Care And Research 45:392-397,2001.

23. Kaya E, Zinnuroglu M, Tugcu I. Kinesio taping compared to physical therapy modalities for the treatment of shoulder impingement syndrome. Clinical rheumatology. 2011 Feb 1;30(2):201-7.

24. Kneeshaw D. Shoulder taping in the clinical setting. Journal of bodywork and movement therapies. 2002 Jan 1;6(1):2-8. 